\title{
Airborne Mapping of Complex Obstacles using 2D Splinegon
}

\author{
Samuel B. Lazarus, Madhavan Shanmugavel, Antonios Tsourdos, Rafał Żbikowski, and Brian A. White
}

\begin{abstract}
This paper describes a recently proposed algorithm in mapping the unknown obstacle in a stationary environment where the obstacles are represented as curved in nature. The focus is to achieve a guaranteed performance of sensor based navigation and mapping. The guaranteed performance is quantified by explicit bounds of the position estimate of an autonomous aerial vehicle using an extended Kalman filter and to track the obstacle so as to extract the map of the obstacle. This Dubins path planning algorithm is used to provide a flyable and safe path to the vehicle to fly from one location to another. This description takes into account the fact that the vehicle is made to fly around the obstacle and hence will map the shape of the obstacle using the 2DSplinegon technique. This splinegon technique, the most efficient and a robust way to estimate the boundary of a curved nature obstacles, can provide mathematically provable performance guarantees that are achievable in practice.
\end{abstract}

Index Terms-Multiple sensor fusion, integrated GPS/INS navigation, Dubins path planning, obstacle avoidance, Splinegon, Boundary estimation, robust estimation.

\section{INTRODUCTION}

The autonomous aerial vehicle has significant advantages over the ground vehicles, where it can achieve a high degree of maneuverability, high speed response time and the ability of large area coverage. Generally the autonomous aerial vehicles carry the required sensors such as inertial sensors (INS), accelerometers and gyroscopes, to measure the acceleration and the angular rate, while the obstacle detection and the map-making is carried out with time of flight sensors such as ultrasonic or laser sensors. The objective of the work described in this paper is to navigate the UAV to desired locations with required orientation so to be able to build the map of its environment. The problems associated with this study are firstly the mapping of complex environments with minimum information, the schedule of the UAV to finite locations (waypoints) and finally the guidance algorithm that can allow the UAV to fly safely from one waypoint to another and control of approach angle to each waypoint.

A nonlinear (Extended) Kalman filter (EKF) is used for the navigation by aiding the measurements from the GPS and INS sensors. The vehicle path is guided by the Dubins path planning algorithm using the circular arcs and straight line segments with the obstacle avoidance system. In order to overcome the problem of data association for the complex environments, an advanced technique is employed in this paper to estimate the boundary of the curved nature obstacles. To illustrate this algorithm of modeling the boundary of a curved nature, the laser sensor measurements are represented as vertices to form a polygon with line segments. The mapping task is achieved by generalisation of a polygon to produce a set of vertices that are connected by line segments of constant curvature. This represents a subset of a class of objects named as splinegons.

Firstly the use of a multiple sensors architecture addressed for robust localisation of the unmanned aerial vehicles, that are flies in an unknown environment with a constant altitude. The localisation is based on the integrated GPS/INS; This robust localisation system increases the stability of the aerial vehicles which in turn provides the sufficient richness in mapping task.

Samuel B. Lazarus, Madhavan Shanmugavel, Antonios Tsourdos, Rafał Żbikowski, and Brian A. White are with the Department of Aerospace, Power \& Sensors, Cranfield University, Shrivenham, Swindon SN6 8LA, United Kingdom.
Secondly the paper addresses a flyable and a safe path of the UAVs using the Dubins path planing algorithm. The Dubins path is the shortest path connecting two poses in a plane with a constraint of maximum curvature. The path planning provides a flyable trajectories to the vehicles to fly from one location to another. Generally, the locations are predefined by the way-points. So, that the vehicle paths are generated by the path planner using the Dubins path planning algorithm. The simple obstacle avoidance system is also a novelty of this paper in which it automatically replans the motion of the vehicle when it approaches close to the obstacle. This in turn provides a stability and a safe path which can protect the vehicles from any collision or leading the vehicles into a catastrophic failure. This proposed guidance algorithm provides terminal angle control which is an important requirement so that the on-board sensor to successfully perform the mapping task.

Finally, the main contribution of this paper is development of an efficient technique called splinegon to build the map of the curvilinear objects. This is a fairly new approach that can handle the data association problem in a simple structure in building the curved nature of the obstacle. The fused EKF estimated positions are used with the limited number of measurements(i.e., the interpolation points) from the laser sensors to build the map. As the vehicle flies around the obstacle in a constant altitude the time of flight sensor such as laser sensor is used to measure the distance with the objects. Out of all these measurements, only a limited number of measurements are chosen that represent the vertices to construct a polygon. The data association algorithm is implemented to select a limited number of vertices and to uniformly distribute these vertices around the obstacle in a reasonable way. This in turn reduces the computational power and the memory usage of the system. This selection of maximum number of vertices that can form the polygon is limited from sixteen to twenty vertices. This selection of vertices are accomplished by calculating the length and the curvatures between each of the vertices and to eliminate the vertex that has a minimum curvature. The selection process is based on the size and the curved nature of the obstacle. Finally, the constructed polygon is generalised to produce a set of vertices that are connected by line segments of constant curvature called splinegon. This splinegon is a set of vertices that have constant curvature line segments defined with $C^{2}$ contact at the vertex points. This splinegon technique gives a solution that has a highly richness in building the curved nature of the unknown obstacle. This proposed approach is a computationally attractive one resulting in guaranteed bounded errors for the fused estimated position in the localisation and mapping of an autonomous aerial vehicle. The development of this algorithm is shown in the functional block diagram in figure 1. As is can be observed in the block diagram, the vehicle path is generated by the path planner which can easily protect the vehicle by applying the obstacle avoidance system. As the vehicle flies the measurement from the GPS sensor is fused with the inertial sensor to estimate the position of the aerial vehicle using an EKF. This is exploited to bound the errors in the inertial sensors which, in turn, gives a better estimated position of the autonomous aerial vehicle, so that, the accuracy and richness in the mapping problem is relatively increased. Hence, a limitation of one sensor is compensated by the other. Finally the splinegon technique is implemented to build the 


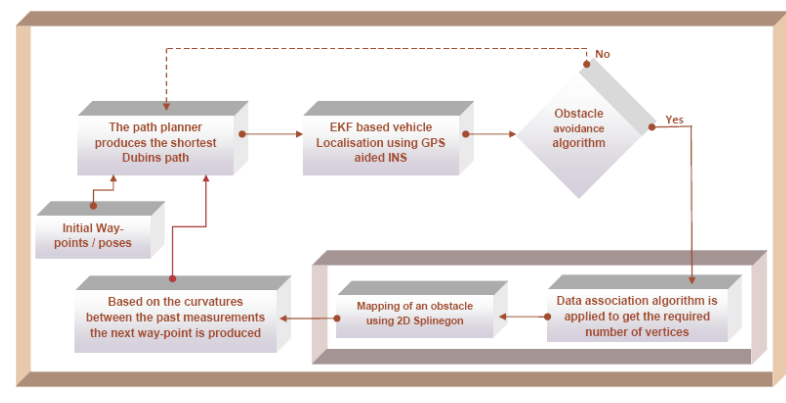

Fig. 1. Functional block diagram of the autonomous aerial vehicle navigation and mapping of a curvilinear objects using 2D splinegon

map of the unknown object.

\section{PRIOR WORK IN THE FIELD}

In recent years there has been a lot of interest in the problem of boundary estimation and boundary tracking by means of robotic networks in particular the problem to design an algorithm that could allow a limited number of sensor swarms to detect the boundary of a static region of interest and estimate it. There are numerous applications such as the detection of harmful algae bloom [1], [2], oil spill [3], and fire boundary estimation [4], [5]. Bertozzi et al.[1] adopts the so called snake algorithm from the computer vision literature to detect and track the boundary of harmful algae bloom. The agents are equipped with a chemical sensor that is able to measure concentration gradient and with a communication system that is able to exchange information with a data fusion centre.

In the paper by Susca and Bullo [6] a group of UAVs optimally place some interpolation points on the boundary of a region of interest. The boundary is then reconstructed by linear interpolation of the interpolation points. The algorithm in [6] is said to be provably convergent as well as in the case of slowly-moving boundaries, because of certain input to state stability properties. The criterion is used to optimally place robots and interpolation points is such that they are uniformly distributed according to a curvature-weighted distance function defined along the boundary. The curvature-weighted distance function is based on the literature on optimal approximation of convex bodies by polygons.

In the field of Localisation and Mapping, Kim and Sukkarieh[7] present an approach for the airborne SLAM using the actual flight and the observation data. The observations are provided by a vision sensor. However, the key limitations of the robustness of these approaches rely only on the underlying mechanism of the image processing where the acquired image is being extracted to get the data; and this processed image is used to determine the current location of the vehicle.

Ning Yang et.al.,[8] proposed a novel particle filter (PF) for sensor fusion, and the sampling importance of resampling particle filter (SIR-PF) is applied to address the nonlinear measurement model. The model showed the better performance with higher accuracy of navigation when compared with the EKF. The simulation results showed that the SIR-PF performed superior over the EKF in the same situations. In addition to that, it is proposed that the PF can be a good substitute for the EKF in on-line land vehicle navigation. Finally the authors have suggested that a new versions of PF with high efficiency and hybrid algorithms based on Kalman filter algorithm should be developed in future.

Frank Dellaert et.al.,[9] investigated the smoothing approaches as a viable alternative to extended Kalman filter (EKF)- based solutions.
An interpretation of factorization in terms of the graphical model associated with the SLAM problem is presented. Both simulation results and actual SLAM experiments in large-scale environments are presented that underscore the potential of these methods as an alternative to EKF-based approaches. Results indicated that the square root information smoothing methods are much better equipped to deal with non-linear process and measurement models than the EKF and yielded the entire smoothed robot trajectory. It recovered the entire trajectory and is exact, and even the sub-optimal incremental scheme we evaluated behaves much better than the EKF as the size of the environment grows.

Given such a large background, this shows that a very few efficient results have been produced in building the map of curvilinear objects.

\section{A. Trajectory generation - Dubins trajectory}

Dubins path is the shortest path connecting two poses for a vehicle flying at a constant altitude with a constraint of maximum curvature [10]. This Dubins path is a composite path of type CLC or CCC or a subset of these two, where ' $C$ ' stands for Circular maneuver and ' $L$ ' stands for straight Line maneuver. In this paper the CLC type trajectories are used. The CLC trajectory comprises two circular maneuvers connected by a tangential straight line maneuver while the CCC trajectory comprises three consecutive tangential circular maneuvers. The subset of these two trajectories falls in the category either CL or LC or CC. The straight line maneuver is the shortest path for a rectilinear motion and the circular maneuver is the shortest trajectory for turning and combined effect of these two maneuvers provides the shortest trajectory for a general motion in $2 D$. Though extensive studies on Dubins trajectory have been carried out by research community, some of the relevant works can be found in [11], [12] and [13] on path planning and [14] on missile applications.

In general, the trajectory generation involves designing a flyable path between two poses or way-points. For a given initial pose $P_{s}\left(x_{s}, y_{s}, \theta_{s}\right)$ and a final pose $P_{f}\left(x_{f}, y_{f}, \theta_{f}\right)$ with constraint on maximum curvature of the vehicle, the trajectory generation involves producing a trajectory $r(t)$ which satisfies the following equation:

$$
P_{s}\left(x_{s}, y_{s}, \theta_{s}\right) \stackrel{r(t)}{\longrightarrow} P_{f}\left(x_{f}, y_{f}, \theta_{f}\right), \kappa(t)<\kappa_{\max }
$$

where $(x, y)$ is the position variable, $\theta$ is the direction/orientation variable and $\kappa_{\max }$ is the maximum curvature or minimum turning radius of the vehicle.

Using the principles of Euclidean geometry, the Dubins trajectory is formed by drawing common tangents (internal \& external) between the circular arcs. Here the design using external tangent is considered. The case of internal tangent is analogous and is omitted for brevity. Refer figure (II-A). The initial and final circular maneuvers are centered respectively at $O_{s}\left(x_{c s}, y_{c s}\right)$ and $O_{f}\left(x_{c f}, y_{c f}\right)$ form two primary circles $C_{s}$ and $C_{f}$. A secondary circle of radius $\left|\rho_{f}-\rho_{s}\right|$ drawn at $O_{f}$ for $\rho_{s} \leq \rho_{f}$. The centers $O_{s}$ and $O_{f}$ are connected by a line $c$. The centers of the primary circles ignoring the direction are:

$$
\begin{gathered}
\left(x_{c s}, y_{c s}\right)=\left(x_{s}-\rho_{s} \cos \left(\theta_{s} \pm \pi / 2\right), y_{s}-\rho_{s} \sin \left(\theta_{s} \pm \pi / 2\right)\right) \\
\left(x_{c f}, y_{c f}\right)=\left(x_{f}-\rho_{f} \cos \left(\theta_{f} \pm \pi / 2\right), y_{f}-\rho_{f} \sin \left(\theta_{f} \pm \pi / 2\right)\right)
\end{gathered}
$$

A perpendicular $O_{f} T_{E N}$ cuts the secondary circle at $T^{\prime}$. Now the $\triangle O_{s} O_{f} T^{\prime}$ forms a right angled triangle with hypotenuse $\left|O_{s} O_{f}\right|$ and other two sides $O_{f} T^{\prime}\left(=\left|\rho_{s}-\rho_{f}\right|\right)$ and $O_{s} T^{\prime}$. The included 


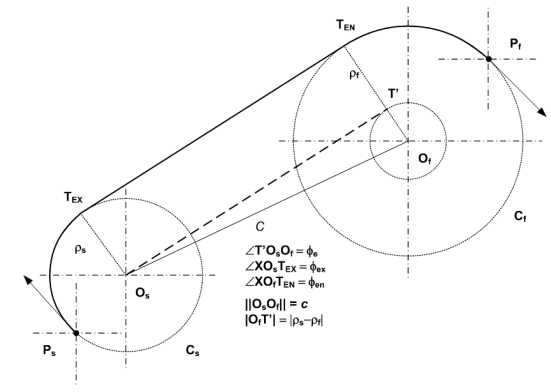

Fig. 2. Dubins - Design of CLC path

angle between $\left|O_{s} O_{f}\right|$ and $O_{s} T^{\prime}$ is $\phi_{e}$ and the slope of the line $c$ is $\psi$.

$$
\begin{array}{r}
\psi=\arctan \left(\frac{y_{c f}-y_{c s}}{x_{c f}-x_{c s}}\right) \\
\phi_{e}=\arcsin \left(\frac{\rho_{f}-\rho_{s}}{c}\right) \\
c=\sqrt{\left(x_{c s}-x_{c f}\right)^{2}+\left(y_{c s}-y_{c f}\right)^{2}}
\end{array}
$$

The line $T_{E X} T_{E N}$ forms the external tangent connecting the two primary circles and is also parallel to $O_{s} T^{\prime}$. The values of the angles $\phi_{e x}\left(=\angle\left(X O_{s} T_{E X}\right)\right)$ and $\phi_{e n}\left(=\angle\left(X O_{f} T_{E N}\right)\right)$ are given in table I. $T_{E X}$ is tangent exit point on starting arc, $T_{E N}$ is the tangent entry

\begin{tabular}{|l|l|c|c|c|}
\hline Start-Turn & Finish-Turn & $\phi_{e}$ & $\phi_{e x}$ & $\phi_{e n}$ \\
\hline Right & Right & $\arcsin \left(\frac{\rho_{f}-\rho_{s}}{c}\right)$ & $\phi_{e}+\frac{\pi}{2}+\psi$ & $\phi_{e}+\frac{\pi}{2}+\psi$ \\
\hline Left & Left & $\arcsin \left(\frac{\rho_{f}-\rho_{s}}{c}\right)$ & $\phi_{e}-\frac{3 \pi}{2}+\psi$ & $\phi_{e}-\frac{3 \pi}{2}+\psi$ \\
\hline
\end{tabular}

\section{TABLE I}

CALCULATION OF TANGENT EXIT AND ENTRY POINTS

point on ending arc. Taking the slope of the connecting line $c$ as $\psi$ the tangent exit and entry points are calculated as below:

$$
\begin{gathered}
T_{E X}=\left(x_{c s}+\rho_{s} \cos \left(\phi_{e x}\right), y_{c s}+\rho_{s} \sin \left(\phi_{e x}\right)\right) \\
T_{E N}=\left(x_{c f}+\rho_{f} \cos \left(\phi_{e n}\right), y_{c f}+\rho_{f} \sin \left(\phi_{e n}\right)\right)
\end{gathered}
$$

All the angles are assumed positive in anticlockwise direction. By connecting the points $P_{s}$ to $T_{E X}$ and $T_{E N}$ to $P_{f}$ respectively by the arc of radii $\rho_{s}$ and $\rho_{f}$, the path from $P_{s}$ through the points $T_{E X}$, $T_{E N}$ and $P_{f}$ forms a CLC Dubins trajectory.

From the figure II-A, for a given pose, there are two circles tangent to it. The pose $P$ have a right turn $r$ on the arc $C_{1}$ and a left turn on the arc $C_{2}$. The centers of the maneuvers are chosen for a given pose either $P_{s}$ or $P_{s}$ by using the equation 1 , the direction of the maneuvers are chosen as per the table I, and the radii of the maneuvers is greater then or equal to the minimum turning radius of the UAVs.

Thus, if either $\theta_{s}$ or $\theta_{f}$ is a free variable, a set of eight paths can be produced. If both the orientations are free variables, a set of sixteen paths can be produced. The shortest path can be selected from the set of available paths.

This path planning algorithm is applied at each iteration which generates the shortest path between the two poses. When the vehicle starts the initial and the final poses are given to generate the first

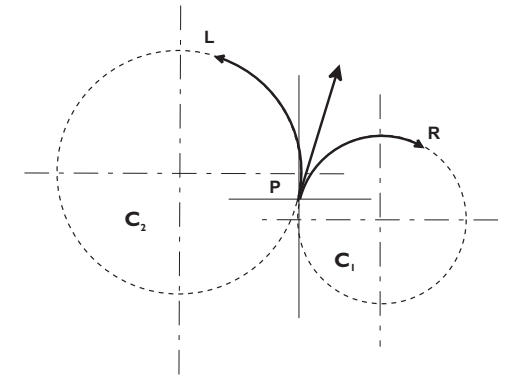

Fig. 3. Tangent Circles

dubins path. As the vehicle moves the next poses or the next waypoints are predicted based on the each of the local updates. So, at each of the iteration a set of new poses is produced to generate the path of the vehicle. In any case, if this generated path leads the vehicle close to any of the obstacles then the obstacle avoidance algorithm is applied to protect the vehicle from a major collision.

\section{B. Obstacle Avoidance}

An obstacle avoidance system is implemented in this project that can protect the flying vehicle from any collision with the surrounded obstacles. This obstacle avoidance algorithm is applied in online while the vehicle is in motion. As the vehicle moves in the path provided by the Dubins the obstacle detection and map making sensor such as laser sensor provides the distance between the vehicle and the surrounded obstacles. If the vehicle is found to be close to any of the obstacles or if the generated Dubins path leads the vehicle towards any obstacles, then the obstacle avoidance algorithm is applied that will automatically re-plan the path of the vehicle in online so as to protect the vehicle from any major collision. In this case the Dubins path planning algorithm requires the current vehicle position with its orientation and the next way-point/poses to re-plan the path of the vehicle. This task is accomplished by choosing a new way-point or generating an intermediate way point which will by pass the obstacle to prevent the collision. This simple algorithm is applied based on the speed and the turning radius of the vehicle. The implementation result while applying this algorithm is shown in figure 4.

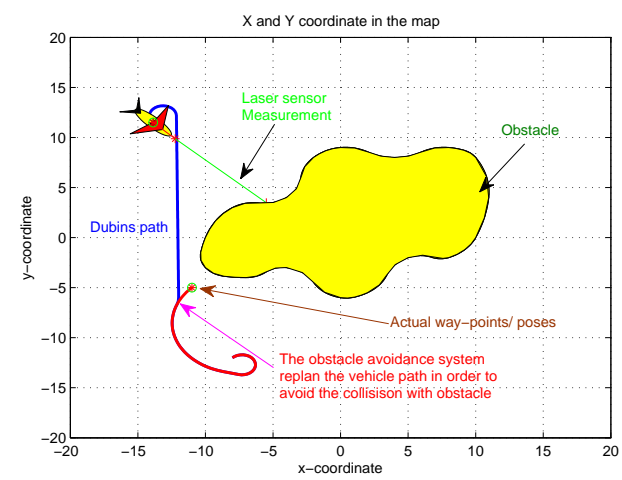

Fig. 4. Obstacle avoidance algorithm

\section{EKF BASED NAVIGATION USING GPS AIDED INS}

In sensor based navigation for an autonomous aerial vehicle, the inertial sensors are often affected by bias, drift and noise. Moreover, by using the inertial sensors (INS) alone the vehicle cannot reduce the errors occurring often in the measurements of the motion with 
INS system. It needs an external information about its absolute position. This can be provided either by GPS or from the known map of the surrounding environment. In this paper a robust estimation algorithm for sensor based navigation is proposed by aiding the GPS measurements with the INS to estimate the vehicle position. In order to accomplish this task the INS sensor measurements are aided with the absolute position provided by the GPS using an extended Kalman filter. So, the use of multiple sensors also has other advantages such as, measurement errors or failure from one of the sensors will not have a catastrophic effect on the system, since the same information will be available from the other sensors. This algorithm is complementary in the sense that they compensate for each other's limitations, so that the resulting performance of the sensor system is better than of its individual components, which in turn, provides more accuracy in the mapping task. A detailed explanation about this GPS aided INS navigation system using Kalman filter is shown in figure 5 .

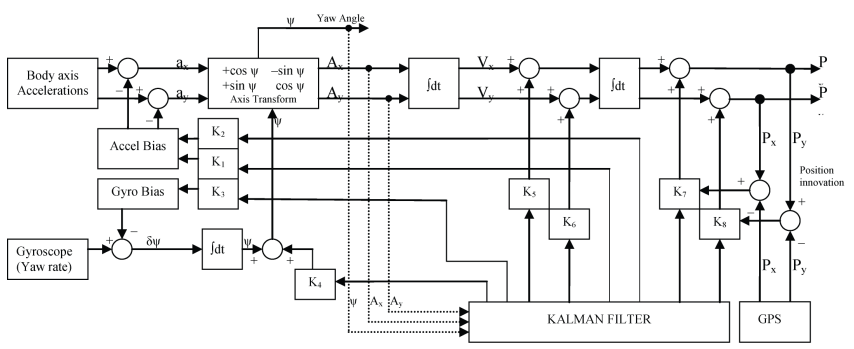

Fig. 5. The block diagram of Kalman filter based autonomous flying vehicle naviagtion using GPS aided INS

\section{MAPPING ThE UNKNOWN CURVED NATURE OBSTACLE USING 2D SPLINEGON}

Mapping the curved nature of the unknown obstacle is modelling the boundary of an obstacle within an urban environment. The obstacle is to be tracked using an aerial vehicle equipped with sensors capable of detecting the boundary of the obstacle. This will enable the measurement points that cloud to be determined to extract the exact shape of the unknown obstacle. As the vehicle moves the time of light sensor such as ultrasonic sensor periodically gives the measurement points. The description will take into account the fact that the vehicle will fly around the obstacle and hence will detect the boundary which is known as the interpolation points. This suggests that the most efficient modelling approach should be to define these measurement points as vertices and these vertices are used to construct a polygon with line segments. Then the constructed polygon is generalised to produce a set of vertices that are connected by line segments of constant curvature. This is a subset of a class of objects named as Splinegons [15],[16] and [17]. To illustrate their use in modelling the boundary of a curved nature a set of vertices that have constant curvature line segments defined with $C^{2}$ contact at the vertex points are considered. The implementation result while applying this method is shown in figure 6 as an example.

The example shown in figure 6 is a splinegon with sixteen vertices with the tangent vectors, together with the arc radii. As required, the radii share a common direction at the vertices, but have different magnitudes. The magnitude is the inverse of the curvature for that segment. Hence two arcs of differing curvature will connect these vertices via the intermediate vertex.

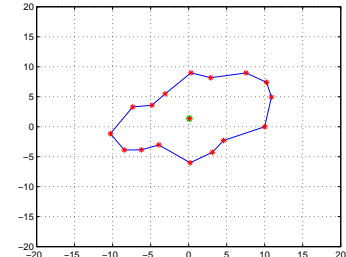

(a) The set of vertices that forms a polygon with line segments

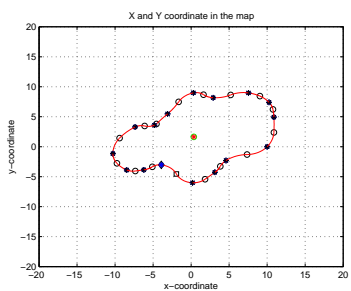

(b) The constructed Splinegon
Fig. 6. splinegon with sixteen vertices- The vertices are shown by the symbol "*" and the intermediate vertex is shown by the symbol "o".

\section{A. 2D Splinegon Construction}

A splinegon with constant curvature line segments can be defined with $C^{2}$ contact at the vertices. This implies that the line segments share both a common vertex and that the tangents at the vertices are identical. In order to ensure $C^{2}$ contact between vertices, the line segments must meet both position and tangent end point constraints. A single arc segment between vertices has only one degree of freedom: the arc curvature. This is not enough to be able to match the tangent constraint at both end vertices, as at least two degrees of freedom are necessary. Extra degrees of freedom are thus required to ensure the $C^{2}$ constraints as both line segment, end vertices can be met. One solution that increases the degrees of freedom is to introduce an intermediate vertex such that the line segment is replaced by two arc segments of different curvature, as shown in figure 7 . The

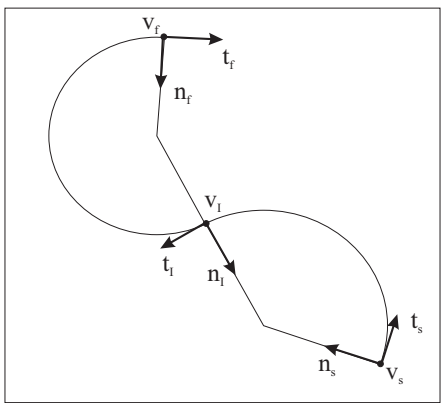

Fig. 7. Arc segment with $C^{2}$ contact intermediate vertex

vertices identified by the vehicle measurement points from the laser sensor are shown by the symbol "*" and the intermediate vertex is shown by the symbol "o". Hence two arcs of differing curvature will connect these vertices via the intermediate vertex. In order to develop the defining equations for such a solution, the intersection of two constant curvature arcs at a point with $C^{2}$ contact is considered. Two orientations for the intermediate arcs are possible. These are shown in figure 8 . Constructing the arc chords and identifying the chord-to-arc angles $\phi_{s}$ and $\phi_{f}$, as shown in figure 8(a), for one orientation of tangent vectors $\boldsymbol{t}_{s}$ and $\boldsymbol{t}_{f}$, and in figure 8(b) for the other orientation, enable the conditions required for $C^{2}$ contact to be defined. The position of the intermediate vertex and the arc curvatures are required to define the composite arc between these vertices. As the conditions for a solution are identical for both orientations, only one will be studied in detail. Hence for orientation 1 shown in figure $8(\mathrm{a})$, summing the angles inside the quadrilateral defined by points 


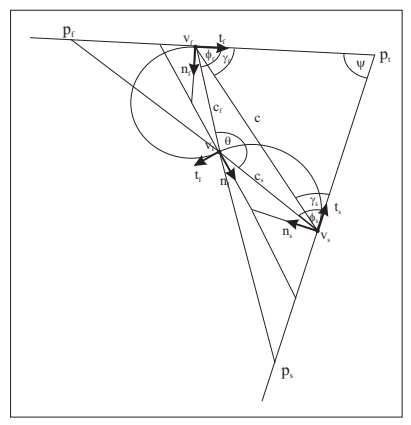
tion 1 (a) $C^{2}$ contact for Orienta-

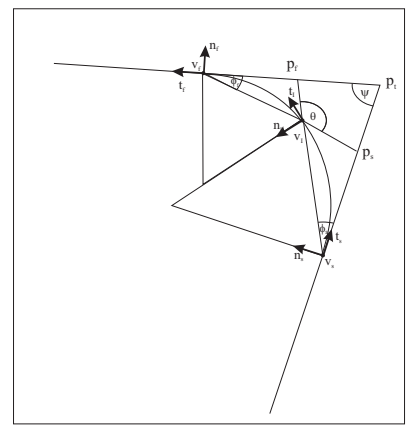

(b) $C^{2}$ contact for Orientation 2
Fig. 8. Chord segments with $C^{2}$ contact

$v_{s}, p_{t}, v_{f}$ and $v_{I}$, the result is:

$$
\theta=2 \pi-\phi_{s}-\phi_{f}-\psi
$$

Figure $8(\mathrm{a})$, using the angle defined by points $v_{s}, v_{I}$ and $v_{f}$, shows the relationship:

$$
\begin{aligned}
\theta & =\pi-\left(\frac{\pi}{2}-\phi_{s}\right)-\left(\frac{\pi}{2}-\phi_{f}\right) \\
& =\phi_{s}+\phi_{f}
\end{aligned}
$$

Hence:

$$
\begin{aligned}
2\left(\phi_{s}+\phi_{f}\right) & =2 \pi-\psi \\
\phi_{f} & =\pi-\frac{\psi}{2}-\phi_{s}
\end{aligned}
$$

Both $\phi_{s} \geq 0$ and $\phi_{f} \geq 0$ are required to maintain consistent arc direction by orienting the directed tangent vector $t_{I}$ at the intermediate vertex $v_{I}$. This is because each arc is directed, in that there is a required transition direction defined by the tangent vectors at these vertices. If $\phi_{s}<0$ and $\phi_{f}<0$ then the arc directions oppose at the intermediate vertex, implying that the arc directions will reverse. This implies that the intermediate tangent vectors for each segment are parallel but have opposite directions. Hence for a consistent solution, the result will be:

$$
\pi-\frac{\psi}{2} \geq \phi_{s} \geq 0
$$

Hence a range of solutions is possible for $\phi_{s}$ and $\phi_{f}$ which will satisfy the bounds in equation (8). Given the vertex chord length $c$ between these vertices $v_{s}$ and $v_{f}$, the arc chord lengths $c_{s}$ and $c_{f}$ can be obtained by using the sine rule on the triangle defined by vertices $v_{s} v_{I}$ and $v_{f}$, as:

$$
\begin{aligned}
c_{s} & =c \frac{\sin \left(\phi_{f}-\gamma_{f}\right)}{\sin (\theta)} \\
c_{f} & =c \frac{\sin \left(\phi_{s}-\gamma_{s}\right)}{\sin (\theta)}
\end{aligned}
$$

where $\gamma_{s}$ and $\gamma_{f}$ are the angles between the vertex tangent vectors $\boldsymbol{t}_{s}$ and $\boldsymbol{t}_{f}$ respectively, and the vertex chord. Using simple geometry, the curvature of each segment can then be determined as:

$$
\begin{aligned}
\kappa_{s} & =\frac{2 \cos \left(\phi_{s} / 2\right)}{c_{s}} \\
\kappa_{f} & =\frac{2 \cos \left(\phi_{f} / 2\right)}{c_{f}}
\end{aligned}
$$

\section{B. Vertex Selection and Data Association}

The splinegon representation assumes some reasonably uniform distribution of vertices around the obstacle. Each vertex will have a curvature and length and these can be used to determine metrics for each segment. The data association association algorithm is implemented here to uniformly distribute the vertices around the obstacle in a reasonable way. Though it seems to be a hard task, it is one of the promising algorithms that fulfils the requirements based on the curvatures of the each vertex. In order to accomplish this task a threshold value for the minimum curvature between any two vertices is chosen. Then the length and the curvatures between each of the vertices are calculated so as to eliminate any vertex that has a minimum length and a curvature lesser than the threshold value. i.e., if the length or the curvature between any of the two vertices is less than or equal to a threshold value then the vertex is eliminated from the list of vertices. This process assures that the given set of vertices can provide a reasonable way to uniformly distribute the vertices around the obstacle to form a polygon. This in turn reduces the computational power of the system and reduces the use of memory by maintaining a selected number or a constant number of vertices throughout the mission. So, this selection process of maximum number of vertices that can form the polygon is limited from sixteen to twenty. The choosing of the threshold valus is an online tunable parameter it can be chosen depending upon the total number of measurements that are obtained at each of the iterations. Geometrically, the number of vertices chosen depends upon the size and the curved nature of the obstacle. This algorithm shows an efficient outcome in limiting the number of vertices to form a polygon.

\section{DISCUSSION AND RESULTS}

In this paper a novel integrated sensor fusion architecture is proposed to achieve a robust airborne navigation and map building that can overcome the uncertainty in the system model and sensor noise statistics. The proposed airborne navigation is based on the integration of GPS and INS which can be implemented for small and micro aerial vehicles in an urban surveillance missions. The complementary position estimates are fused, thereby resulting in a much improved position estimate from the EKF which will increase the accuracy and richness in the mapping task.

An advanced technique that can provide a sufficient richness in mapping the curved nature of an obstacle is proposed. This proposed algorithm along with the Dubins path to generate the path of the autonomous vehicle is robust enough to handle the collision avoidance that can provide the safe and stable path so as to accomplish the mission. The data association algorithm is used to extract the shape of the curved nature of the obstacle in a simple structure which uses a limited or a constant number of vertices to construct the polygon. Finally this constructed polygon, generalised with line segments with a constant curvatures, is called 2D splinegon. This proposed algorithm is implemented for a different static environment which contains both the concave and convex shaped obstacles. The implementation results while applying this algorithm are shown in figures 9 and 10. As can be observed from these figures, At each of these iteration the measurement points that are obtained from the laser sensor are processed with the data association algorithm where it will select the required number of vertices to form a polygon. At the end of the mission this selection of vertices are chosen based on the shape of the obstacle produced in the previous iteration. Finally the list of selected vertices with the line segments are generalised to get the shape of the curved nature of the obstacle using the 2D splinegon technique while the EKF base navigation is applied periodically to 


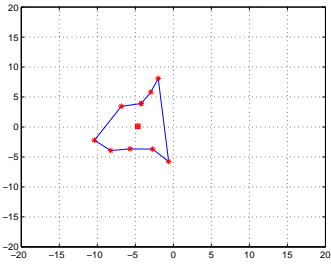

(a) The set of vertices that forms a polygon with line segments

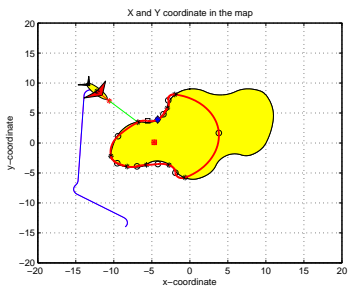

(b) The constructed Splinegon
Fig. 9. The local updated polygon and the map using Splinegon at each cycles

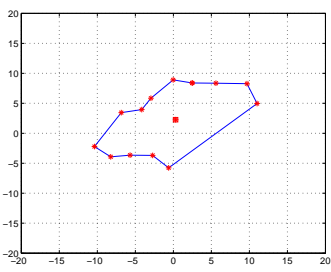

(a) The set of vertices that forms a polygon with line segments

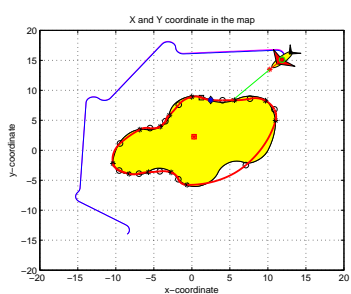

(b) The constructed Splinegon
Fig. 10. The local updated polygon and the map using Splinegon at each cycles

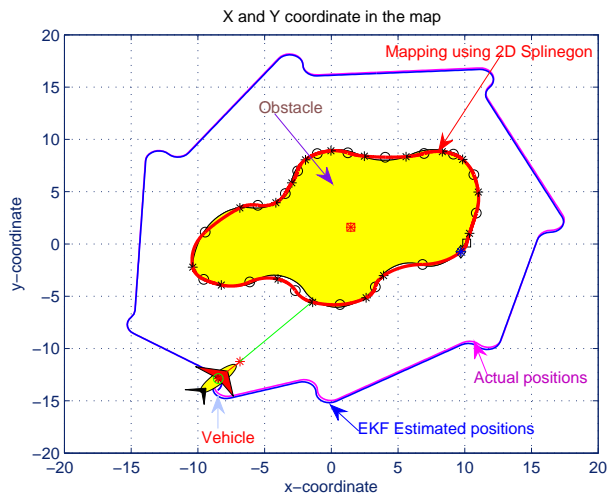

Fig. 11. Airborne Mapping using 2D Splinegon

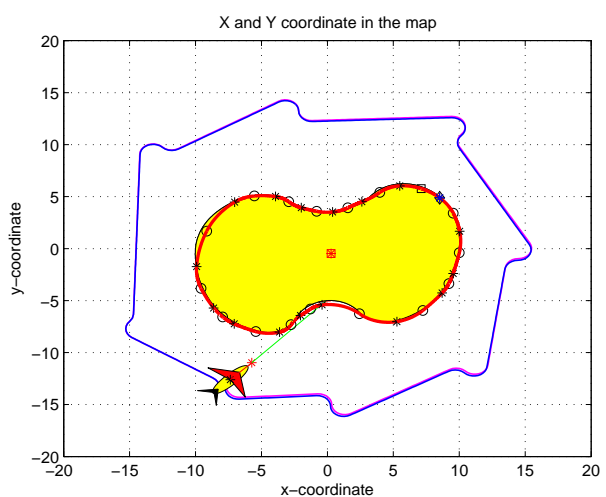

Fig. 12. Airborne Mapping using 2D Splinegon navigate the vehicle. The global updated implementation results are shown in figures $11 \& 12$.

It is proposed to extend this work for a multiple platform with a multiple airborne vehicle that would make the better resulting performance in mapping task. This proposed method, the most recent approach, will result in the sufficient richness in the mapping task which will increase the confidence in boundary estimation thereby decreasing the instability of the (i.e. decreasing the uncertainty) autonomous aerial vehicle.

\section{REFERENCES}

[1] D. Marthaler and A. L. Bertozzi. Tracking environmental level sets with autonomous vehicles. In Proceedings of the Conference on Cooperative Control and Optimization, Gainesville, FL, Dec, December 2002.

[2] A. L. Bertozzi, M. Kemp, and D. Marthaler. Determining environmental boundaries: asynchronous communication and physical scales. In V. Kumar, N. E. Leonard, and A. S. Morse, editors, Proceedings of the 2003 Block Island Workshop on Cooperative Control, volume 309 of Lecture Notes in Control and Information Sciences, pages 25-42, New York, 2004. Springer Verlag.

[3] J. Clark and R. Fierro. Cooperative hybrid control of robotic sensors for perimeter detection and tracking. In American Control Conference, pages 3500-3505, Portland, OR, June 2005.

[4] D. W. Casbeer, S.-M. Li, R. W. Beard, R. K. Mehra, and T. W. McLain. Forest fire monitoring with multiple small uavs. In American Control Conference, pages 3530-3535, Portland, OR, June 2005.

[5] D. W. Casbeer, D. B. Kingston, R. W. Beard, T. W. Mclain, and S.-M. Li. Cooperative forest fire surveillance using a team of small unmanned air vehicles. International Journal of Systems Sciences, 2005.

[6] S. Susca, S. Martnez, and F. Bullo. Monitoring environmental boundaries with a robotic sensor network. Technical report, Center for Control, Dynamical Systems and Computation, University of California at Santa Barbara, Santa Barbara, CA, 93106-5070, USA, March 2006.

[7] Jong-Hyuk Kim and Salah Sukkarieh. Airborne simultaneous localisation and map building. Proceedings of the 1003 IEEE lnternational Conference on Robotics \& Automation, September 2006.

[8] Ning Yang, Wei Feng Tian, Zhi Hua Jin, and Chuan Bin Zhang. Particle filter for sensor fusion in a land vehicle navigation system. Measurement Science and Techonology, 16:677681, 2005.

[9] Frank Dellaert and Michael Kaess. Square root sam: Simultaneous localization and mapping via square root information smoothing. The International Journal of Robotics Research, 25(12):1181-1203, December 2006.

[10] L.E.Dubins. On curves of minimal length with a constraint on average curvature and with perscribed initial and terminal positions and tangent. American Journal of Mathematics, 79:497-516, 1957.

[11] J. J. Enright and E. Frazzoli. On multiple uav routing with stochastic targets: Performance bounds and algorithms. In AIAA Guidance, Navigation, and Control Conference and Exhibit, San Francisco, California, 15 - 18 August 2005.

[12] M. Shanmugavel. Path Planning of Multiple Autonomous Vehicles. PhD thesis, Cranfield University, 2007.

[13] Ketan Savla, Emilio Frazzoli, and Francesco Bullo. On the dubins traveling salesperson problems: novel approximation algorithms. In Robotics: Science and Systems II. MIT Press, Cambridge, MA, August 2006.

[14] M. Robb, B. White, and A. Tsourdos. Earliest intercept line guidance: A novel concept for improving mid-course guidance in area air defence. AIAA Guidance, Navigation and Control Conference and Exhibit, San Francisco, California, Aug. 15-18 2005.

[15] D. P. Dobkin, D. L. Souvaine, and C. J. Van Wyk. Decomposition and intersection of simple splinegons. Algorithmica, 3:473-485, 1988.

[16] D. P. Dobkin and D. L. Souvaine. Computational geometry in a curved world. Algorithmica, 5(3):421-457, 1990.

[17] J. F. Garten, C. E. Schemm, and A. R. Croucher. Modeling the transport and dispersion of airborne contaminants: A review of techniques and approaches. Johns Hopkins APL technical digest, 24(4):368-375, 2003. 